\title{
CORRECTION
}

\section{Correction to: Withholding or withdrawing of life-sustaining therapy in older adults ( $\geq 80$ years) admitted to the intensive care unit}

\author{
Bertrand Guidet ${ }^{1,2^{*}} \mathbb{0}$, Hans Flaatten ${ }^{3,4}$, Ariane Boumendil ${ }^{1 *}$, Alessandro Morandi ${ }^{5,6}$, Finn H. Andersen ${ }^{7,8}$, \\ Antonio Artigas ${ }^{9}$, Guido Bertolini ${ }^{10}$, Maurizio Cecconi ${ }^{11}$, Steffen Christensen ${ }^{12}$, Loredana Faraldi ${ }^{13}$, \\ Jesper Fjølner ${ }^{12}$, Christian Jung ${ }^{14}$, Brian Marsh ${ }^{15}$, Rui Moreno ${ }^{16}$, Sandra Oeyen ${ }^{17}$, Christina Agwald Öhman ${ }^{18}$, \\ Bernardo Bollen Pinto ${ }^{19}$, Ivo W. Soliman ${ }^{20}$, Wojciech Szczeklik ${ }^{21}$, Andreas Valentin ${ }^{22}$, Ximena Watson ${ }^{11}$, \\ Tilemachos Zafeiridis ${ }^{23}$ and Dylan W. De Lange ${ }^{20}$ on behalf of The VIP1 Study Group
}

๑ 2018 Springer-Verlag GmbH Germany, part of Springer Nature and ESICM

\section{Correction to: Intensive Care Med} https://doi.org/10.1007/s00134-018-5196-7

In the original publication Dr Patrick Meybohm of the Department of Anaesthesiology, Intensive Care Medicine and Pain Therapy, Frankfurt University Hospital, Frankfurt, Germany was inadvertently omitted from the list of investigators.

Moreover, there were errors in the listing of the following investigators:

Bernardo Bollen Pinto, Peri-interventional Intermediate Care (SINPI), Geneva University Hospitals, Switzerland.

Paul Abraham, Adult Intensive Care (SIA), Geneva University Hospitals, Switzerland.

Leila Hergafi, Service des Soins intensifs, Hôpital fribourgeois, Fribourg, Switzerland.

*Correspondence: bertrand.guidet@aphp.fr;

ariane.boumendil@gmail.com

${ }^{1}$ Hôpitaux de Paris, Hôpital Saint-Antoine, service de réanimation médicale, 184 rue du Faubourg Saint Antoine, 75012 Paris, France

Full author information is available at the end of the article

The VIP-LST collaborators are listed in the Acknowledgements and in the Electronic Supplementary Material.

The original article can be found online at https://doi.org/10.1007/s0013 4-018-5196-7.
Joerg C. Schefold, Universitätsklinik für Intensivmedizin, Inselspital, Bern University Hospital, University of Bern, Switzerland.

Ewelina Biskup, Medical ICU, University Hospital Basel, Switzerland.

Petr Piza, KARIP, IKEM, Czech Republic.

The lead authors regret these mistakes and apologize for any inconvenience caused.

\section{Electronic supplementary material}

The online version of this article (https://doi.org/10.1007/s00134-018-5312-8) contains supplementary material, which is available to authorized users.

\footnotetext{
Author details

${ }^{1}$ Hôpitaux de Paris, Hôpital Saint-Antoine, service de réanimation médicale, 184 rue du Faubourg Saint Antoine, 75012 Paris, France. ${ }^{2}$ Institut Pierre Louis d'Epidémiologie et de Santé Publique, Sorbonne Universités, UPMC Univ Paris 06, INSERM, UMR_S 1136, 75013 Paris, France. ${ }^{3}$ Department of Clinical Medicine, University of Bergen, Bergen, Norway. ${ }^{4}$ Department of Anaesthesia and Intensive Care, Haukeland University Hospital, Bergen, Norway. ${ }^{5}$ Department of Rehabilitation, Hospital Ancelle di Cremona, Cremona, Italy. ${ }^{6}$ Geriatric Research Group, Brescia, Italy. ${ }^{7}$ Department of Anaesthesia and Intensive Care, Ålesund Hospital, Ålesund, Norway. ${ }^{8}$ Department of Circulation and Medical Imaging, NTNU, Trondheim, Norway. ${ }^{9}$ Department of Intensive Care Medicine, CIBER Enfermedades Respiratorias, Corporacion Sanitaria Universitaria Parc Tauli, Autonomous University of Barcelona, Sabadell, Spain. ${ }^{10}$ Laboratorio di Epidemiologia Clinica, Centro di Coordinamento GiViTI Dipartimento di Salute Pubblica, IRCCS - Istituto di Ricerche Farmacologiche "Mario Negri" Villa Camozzi, 24020, Ranica, Bergamo, Italy. ${ }^{11}$ St George's University Hospital, London, UK. ${ }^{2}$ Department of Anaesthesia and Intensive Care Medicine, Aarhus University Hospital, Åarhus, Denmark. ${ }^{13}$ Grande Ospedale Metropolitano
} 
Niguarda, Milan, Italy. ${ }^{14}$ Department of Cardiology, Pulmonology and Angiology, University Hospital, Düsseldorf, Germany. ${ }^{15}$ Mater Misericordiae University Hospital, Dublin, Ireland. ${ }^{16}$ Unidade de Cuidados Intensivos Polivalente Neurocríticos, Hospital de São José, Centro Hospitalar de Lisboa Central, Faculdade de Ciência Médicas de Lisboa, Nova Médical School, Lisbon, Portugal. ${ }^{17}$ Department of Intensive Care 1K12IC, Ghent University Hospital, Ghent, Belgium. ${ }^{18}$ Karolinska University Hospital, Solna, Sweden. ${ }^{19}$ Geneva University Hospitals, Geneva, Switzerland. ${ }^{20}$ Department of Intensive Care, University Medical Center, University Utrecht, Utrecht, The Netherlands. ${ }^{21}$ Intensive Care and Perioperative Medicine Division, Jagiellonian University Medical College, Kraków, Poland. ${ }^{22}$ Kardinal Schwarzenberg Hospital, Schwarzach, Austria. ${ }^{23}$ Intensive Care Unit General Hospital of Larissa, Larissa, Greece.

\section{Acknowledgements}

VIP1 Study Group members: René Schmutz, B5, Hospital of St. John of God Vienna, Austria; Franz Wimmer, Interne Intensiv, Kardinal

Schwarzenberg'sches Krankenhaus, Austria; Philipp Eller, Intensivstation der Univ--Klinik für Innere Medizin, Medical University Graz, Austria; Klemens Zotter, MICU, University Hospital Innsbruck, Austria; Pieter De Buysscher, Department of Intensive Care, AZ Sint-Lucas Ghent, Belgium; Nikolaas De Neve, Department of Intensive Care, O.L.Vrouwhospital Aalst, Belgium; Sandra Oeyen, Department of Intensive Care, Ghent University Hospital, Belgium; Walter Swinnen, Department of Intensive Care Medicine, AZ Sint-Blasius Dendermonde, Belgium; loannis Taliadoros, CY001, Nicosia General Hospital, Cyprus, Cyprus; Petr Piza, KARIP, IKEM, Czech Republic; Jesper Fjølner, ITA, Randers Regional Hospital, Denmark; Nilanjan Dey, Intensiv Herning, Regions Hospital Herning, Denmark; Christoffer Sølling, I-25, Regionshospital Viborg, Denmark; Bodil Steen Rasmussen, ICU, Aalborg University Hospital, Denmark; Steffen Christensen, OPI Ost, Aarhus University Hospital Skejby, Denmark; Xavier Forceville, Réanimation médico chirurgical, Centre Hospitalier de Meaux, France; Guillaume Besch, Département d’Anesthésie Réanimation Chirurgicale, Centre Hospitalier Régional Universtaire de Besançon, France; Herve Mentec, Service de Réanimation Polyvalente, Centre Hospitalier Victor Dupouy Argenteuil, France; Philippe Michel, Réanimation médico-chirurgicale, CH Carnelle - Portes de l'Oise, France; Philippe Mateu, Réanimation Polyvalente, $\mathrm{CH}$ de Charleville-Mézières, France; Philippe Michel, Réanimation médico-chirurgicale, CH René Dubos, France; Lucie Vettoretti, Réanimation Médicale, CHRU de Besançon, France; Jeremy Bourenne, Reanimation des Urgences et Médicale, CHU Marseille - Timone, France; Nathalie MARIN, reanimation médicale, hopital cochin, France; Max Guillot, Réanimation médicale, Hôpital de Hautepierre, France; Naida Aissaoui, Réanimation médicale, hopital européen georges pompidou, France; Cyril Goulenok Réanimation Médicale, Hopital Privé Jacques CARTIER, France; Nathalie Thieulot-Rolin , Intensive care medicine department, Hospital Marc Jacquet 77000 Melun, France; Jonathan Messika, Réanimation Médico-Chirurgicale, Louis Mourier, France; Lionel Lamhaut, Polyvalente adult ICU, Necker (APHP), France; Bertrand Guidet, Réanimation médicale, Saint Antoine, France; Cyril Charron, Medical-surgical ICU, University Hospital Ambroise Paré, de Paris Boulogne-Billancourt, France, France; Alexander Lauten, 1) Department of Cardiology, 2) DZHK Berlin partner side, Charité Universitaetsmedizin Berlin, Germany; Anna Lena Sacher, Department of Anesthesiology, Charité Universitaetsmedizin Berlin, Germany; Thorsten Brenner, Department of Anesthesiology, Heidelberg University Hospital, Germany; Marcus Franz, Department of Internal Medicine, Jena University Hospital, Friedrich-Schiller University, Germany; Frank Bloos, Department of Anesthesiology, Jena University Hospital, Friedrich-Schiller University, Germany; Henning Ebelt, Department for Medicine II, Catholic Hospital "St. Johann Nepomuk", Germany; Stefan J Schaller, Department of Anesthesiology, Klinikum rechts der Isar, Technical University of Munich, Munich, Germany, Germany; Kristina Fuest, Department of Anesthesiology, Klinikum rechts der Isar, Technical University of Munich, Munich, Germany, Germany; Christian Rabe, Dept. Of Clinical Toxicology, Klinikum rechts der Isar, Technical University of Munich, Munich, Germany, Germany; Thorben Dieck, Department of Anaesthesiologyand Intensive Care, Medical School Hospital Hannover, Germany; Stephan Steiner, Department of Cardiology, Pneumology and Intensive Care, St. Vincenz Krankenhaus Limburg, Germany; Tobias Graf, Department of Cardiology, University Heart Center Luebeck, Germany; Amir M Nia, Division of Cardiology and Intensive Care, University Hospital Düsseldorf, Heinrich-Heine University, Germany; Christian Jung, Division of Cardiology and Intensive Care, University Hospital Düsseldorf, Heinrich-Heine University, Germany; Rolf Alexander Janosi, Department of Cardiology and Vascular Diseases, University Hospital
Essen, Germany; Philipp Simon, Department of Anaesthesiology and ICM, University Hospital of Leipzig, Germany; Stefan Utzolino, Department of General and Visceral Surgery, Universitätsklinikum Freiburg, Germany; Tim Rahmel, Department of Anaesthesiology, Intensive Care Medicine, University Hospital Knappschaftskrankenhaus Bochum, Germany; Eberhard Barth, Department of Anaesthesiology, University of Ulm, Germany; Christian Jung, , University Hospital Düsseldorf, Heinrich-Heine-University Düsseldorf, Medical Faculty, Division of Cardiology, Pulmonology and Vascular Medicine, Düsseldorf, Germany, Germany; Michael Schuster, Department of Anaesthesiology, University Hospital Mainz, Germany; Patrick Meybohm, Department of Anaesthesiology, Intensive Care Medicine and Pain Therapy, , Frankfurt University Hospital, Frankfurt, Germany; Zoi Aidoni , ICU, UGHT AHEPA, Greece; Stavros Aloizos, ICU , Army Share Fund Hospital, Athens, Greece; Polychronis Tasioudis , ICU, G. Gennimatas hospital of Thessaloniki, Greece; Kleri Lampiri , ICU, General Hospital Of Kavala, Greece; Vasiliki Zisopoulou, ICU1, General Hospital Of Larissa, Greece; Ifigenia Ravani , ICU, General hospital of Patras, Greece; Eumorfia Pagaki , ICU, General hospital of Trikala, Greece; Angela Antoniou , ICU, Volos General Hospital, Greece; Theodoros A. Katsoulas, ICU, "Ag Anargyroi" General Hospital, Greece; Aikaterini Kounougeri , ICU , Konstantopouleion General Hospital, Athens, Greece; George Marinakis, ICU, "Korgialenio-Benakio" G. Hospital of Athens, Greece; Fotios Tsimpoukas, ICU, Lamia General Hospital, Greece; Anastasia Spyropoulou, ICU, Panarkadian General Hospital of Tripolis, Greece; Paris Zygoulis, General ICU, University hospital of Larisa, Greece; Aikaterini Kyparissi, ICU, "HIPPOCRATEIO" General Hospital of Athens, Greece; Manish Gupta, MICU, MAX SUPER SPECIALTY HOSPITAL, Vaishali, India; Mohan Gurjar, Department of Critical Care Medicine, Sanjay Gandhi Postgraduate Institute of Medical Sciences, India; Ismail M Maji , MICU, ST JOHNS MEDICAL COLLEGE HOSPITAL, Bangaluri, India; Ivan Hayes, CUH GICU, Cork University Hospital, Ireland; Brian Marsh, Department of Critical Care Medicine, Mater Misericordiae University Hospital, Ireland; Yvelynne Kelly, General ICU, St. Jamess Hospital, Ireland; Andrew Westbrook, ICU, St. Vincents University Hospital, Ireland; Gerry Fitzpatrick, Tallaght intensive Care, Tallaght Hospital, Ireland; Darshana Maheshwari, UHG ICU, University hospital galway, Ireland; Catherine Motherway, ICU, University Hospital limerick, Ireland; Giovanni Negri, Rianimazione, A.S.S.T. Ovest Milanese - Presidio Di Magenta - Ospedale G. Fornaroli (Magenta), Italy; Savino Spadaro, Unità di Terapia Intensiva del Servizio di Anestesia, Azienda Ospedaliera Universitaria Sant Anna (Ferrara), Italy; Giuseppe Nattino, Rianimazione generale, ASSTLecco - Ospedale A.Manzoni (Lecco), Italy; Matteo Pedeferri, Rianimazione, AO della Provincia di Lecco - Presidio Ospedaliero S.Leopoldo Mandic, Merate, Italy; Annalisa Boscolo, Giustiniani I e II (Istar), Azienda Ospedaliera di Padova (Padova), Italy; Simona Rossi, Servizio Anestesia Rianimazione, Azienda Ospedaliera G.Salvini - Presidio Ospedaliero di Rho, Italy; Giuseppe Calicchio, Centro di Rianimazione, Azienda Ospedaliera Universitaria San Giovanni di Dio e Ruggi d'Aragona, Italy; Lucia Cubattoli, Rianimazione Generale, Azienda Ospedaliera Universitaria Senese (Siena), Italy; Gabriella Di Lascio, Terapia Intensiva di Emergenza, Azienda Ospedaliero Universitaria Careggi (Firenze), Italy; Maria Barbagallo, UO 2 Anestesia Rianimazione Terapia Intensiva, Azienda Ospedaliero-Universitaria di Parma (Parma), Italy; Francesco Berruto, rianimazione, Ospedale E. Agnelli (Pinerolo), Italy; Daniela Codazzi, UnitàTerapia Intensiva, Fondazione IRCCS Istituto Nazionale dei Tumori (Milano), Italy; Andrea Bottazzi, Rianimazione 2, Fondazione IRCCS Policlinico S.Matteo (Pavia), Italy; Paolo Fumagalli, Rianimazione 1, Fondazione Policlinico San Matteo (Pavia), Italy; Giancarlo Negro, Anestesia e Rianimazione 1, Ospedale Francesco Ferrari (Casarano), Italy; Giuseppe Lupi, Servizio Anestesia e Rianimazione, Ospedale Maggiore (Cremona), Italy; Flavia Savelli, Anestesia e Rianimazione - TI 2, Ospedale Maurizio Bufalini (Cesena), Italy; Giuseppe A. Vulcano, Terapia Intensiva, Ospedale Civile Nicola Giannettasio (Rossano), Italy; Roberto Fumagalli, Anestesia e Rianimazione 1, Ospedale Niguarda Ca'Granda (Milano), Italy; Andrea Marudi, Rianimazione Neurorianimazione, Nuovo Ospedale Civile Sant Agostino Estense (Modena), Italy; Ugo Lefons, Terapia intensiva, Ospedale Alta Val d'Elsa (Poggibonsi), Italy; Rita Lembo, Rianimazione generale, Ospedale Castelli di Verbania (Verbania), Italy; Maria Babini, Servizio Anestesia e Rianimazione, Ospedale Civile Lugo (Lugo), Italy; Alessandra Paggioro, Struttura Semplice di Rianimazione e Terapia Intensiva, Ospedale degli Infermi di Biella - ASL BI (Biella), Italy; Vieri Parrini, Anestesia e Rianimazione, Ospedale del Mugello (Borgo San Lorenzo), Italy; Maria Zaccaria, Rianimazione e Terapia Intensiva, Ospedale di Ciriè (Torino), Italy; Stefano Clementi, terapia intensiva polivalente, Ospedale di Sesto San Giovanni (Sesto San Giovanni), Italy; Carmelo Gigliuto, Rianimazione, Ospedale di Vigevano - Azienda Ospedaliera 
della Provincia di Pavia (Vigevano), Italy; Francesca Facondini, Reparto di Rianimazione e Terapia Intensiva, Ospedale Infermi (Rimini), Italy; Simonetta Pastorini, Servizio Anestesia-Rianimazione, Ospedale P. Cosma-AUSL 15 Alta padovana (Camposampiero), Italy; Susanna Munaron, Unità di Terapia Intensiva, Ospedale San Giacomo (Castelfranco Veneto), Italy; Italo Calamai, Rianimazione, Ospedale San Giuseppe (Empoli), Italy; Anna Bocchi, Terapia Intensiva, Ospedale San Luca (Trecenta), Italy; Adele Adorni, Unitàdi Terapia Intensiva Rianimatoria, Ospedale Valduce (Como), Italy; Maria Grazia Bocci, Centro di Rianimazione,Policlinico Agostino Gemelli (Roma), Italy; Andrea Cortegiani, Unità di Terapia Intensiva Polivalente, Policlinico P. Giaccone. University of Palermo, Italy; Tiziana Casalicchio, Terapia Intensiva, Ospedale San Giovanni Bosco (Torino), Italy; Serena Mellea, Unità di Terapia Intensiva, Ospedale Santa Maria della Misericordia (Perugia), Italy; Elia Graziani, UnitàOperativa Anestesia e Rianimazione, Santa Maria delle Croci (Ravenna), Italy; Massimo Barattini, Rianimazione, Ospedale Santa Maria Nuova (Firenze), Italy; Elisabetta Brizio, Servizio di Rianimazione, Ospedale SS Annunziata, Italy; Maurizio Rossi, UO Anestesia e Rianimazione, Azienda Ospedaliera Sant'Anna Como - Presidio di Menaggio, Italy; Michael Hahn, ICU, Haugesund hospital, Norway; Hans Flaatten, General ICU, Haukeland University Hospital, Norway; Nicolai Kemmerer, ICU, Kongsberg hospital, Norway; Hans Frank Strietzel, ICU, Kristiansund Hospital, Norway; Knut Dybwik, ICU, Nordlandssykehuset Bodo, Norway; Terje Legernaes, ICU, Hamar hospital, Norway; Pål Klepstad, Dept Intensive Care Medicine, St Olavs University Hospital, Norway; Even Braut Olaussen, ICU, Stavanger University Hospital, Norway; Knut Inge Olsen, ICU, Namsos Hospital, Norway; Ole Marius Brresen, ICU, Telemark Hospital, Skien, Norway; Geir Bjorsvik, ICU , University Hospital Tromso, Norway; Finn H. Andersen, ICU, Ålesund hospital, Norway; Sameer Maini, Medical ICU, Aalesund Hospital, Norway; Lutz Fehrle, ICU, Molde hospital, Norway; Miroslaw Czuczwar, ICU, First Public Teaching Hospital in Lublin, Poland; Pawel Krawczyk, ICU, University Hospital in Krakow, Poland; Mirosław Ziętkiewicz, Respiratory and Thoracic Surgical ICU, John Paul II Hospital in Krakow, Poland; Łukasz R. Nowak, Department of Anaesthesiology and Intensive Care, Maria Sklodowska-Curie Memorial Institute of Oncology Krakow Branch, Poland; Katarzyna Kotfis, Department of Anaesthesia, Intensive Care and Acute Poisonings, Pomeranian Medical University, Teaching Hospital No.2, Poland; Katarzyna Cwyl, ICU, RCZ in Lublin, Poland; Ryszard Gajdosz, Department of Anaesthesiology and Intensive Care, Scanmed St. Raphael Hospital in Kraków, Poland; Jowita Biernawska, Department of Anaesthesiology and Intensive Care, Pomeranian Medical University, Teaching Hospital No. 1, Poland; Romuald Bohatyrewicz, Department of Anaesthesiology and Intensive Care, Pomeranian Medical University, Teaching Hospital No. 1, Poland; Ryszard Gawda, Department of Anaesthesiology and Intensive Care, University Hospital in Opole, Poland; Paweł Grudzień, ICU, Edward Szczeklik Specialist Hospital in Tarnów, Poland; Paweł Nasiłowski, Department of Anaesthesiology and Intensive Care, Gabriel Narutowicz Specialist Hospital in Kraków, Poland; Natalia Popek, Department of Anaesthesiology and Intensive Care, Stefan Żeromski Specialist Hospital in Kraków, Poland; Waldemar Cyrankiewicz, Department of Anaesthesiology and Intensive Care, Antoni Jurasz University Hospital No. 1 in Bydgoszcz, Poland; Katarzyna Wawrzyniak, Department of Anaesthesiology and Intensive Care, Antoni Jurasz University Hospital No. 1 in Bydgoszcz, Poland; Marek Wnuk, Department of Anaesthesiology and Intensive Care, John Paul II Memorial Hospital in Bełchatów, Poland; Dariusz Maciejewski, Department of Anaesthesiology and Intensive Care, Provincial hospital in Bielsko-Biała, Poland; Dorota Studzińska, Department of Anaesthesiology and Intensive Care, St. John Grande Hospital, Poland; Maciej Żukowski, Department of Anaesthesiology, Intensive Care and Acute Poisoning, Pomeranian Medical University, Teaching Hospital No. 2 in Szczecin, Poland; Szymon Bernas, Department of Anaesthesiology and Intensive Therapy Centre for Artificial Extracorporeal Kidney and Liver Support, Dr Władysław Biegański Regional Specialist Hospital in Łódź, Poland; Mariusz Piechota, Department of Anaesthesiology and Intensive Therapy Centre for Artificial Extracorporeal Kidney and Liver Support, Dr Władysław Biegański Regional Specialist Hospital in Łódź, Poland; , Centre for Artificial Extracorporeal Kidney and Liver Support, , Poland; Wojciech Szczeklik, Department of Intensive Care and Perioperative Medicine, University Hospital in Kraków, Poland; Ilona Nowak, Department of Intensive Care and Perioperative Medicine, University Hospital in Kraków, Poland; Jakub Fronczek, Department of Intensive Care and Perioperative Medicine, University Hospital in Kraków, Poland; Marta Serwa, Department of Anaesthesiology and Intensive Care, University Hospital and Educational Centre of the Medical University of Łódź, Poland; Waldemar Machała, Department of Anaesthesiology and Intensive
Care, University Hospital and Educational Centre of the Medical University of Łódź, Poland; Jan Stefaniak, Department of Anaesthesiology and Intensive Care, University Clinical Centre in Gdańsk, Poland; Maria Wujtewicz, Department of Anaesthesiology and Intensive Care, University Clinical Centre in Gdańsk, Poland; Paweł Maciejewski, Department of Anaesthesiology and Intensive Care, Orthopedic-Rehabilitation University Hospital in Zakopane, Poland; Małgorzata Szymkowiak, Department of Anaesthesiology and Intensive Care, Józef Struś Hospital in Poznan, Poland; Barbara Adamik, Department of Anaesthesiology and Intensive Care, Wrocław University Hospital, Poland; Nuno Catorze, UCIP, C. H. Médio TEJO, Portugal; Miguel Castelo Branco, Unidade de Cuidados Intensivos, Centro Hospitalar Cova da Beira, EPE, Portugal; Inês Barros, Unidade de Cuidados Intensivos Polivalente, Centro Hospitalar Tondela-Viseu, Portugal; Nelson Barros, Serviço Medicina Intensiva, Centro Hospitalar Trás-os-Montes e Alto Douro, Portugal; Andriy Krystopchuk, Intensive Care and Emergency Department, Centro Hospitalar do Algarve-Hospital de Faro, Portugal; Teresa Honrado, Unidade Cuidados INtensivos Polivalente, Hospital de São João, Portugal; Cristina Sousa, UCI, Hospital da Luz, Portugal; Francisco Munoz, UMI, Hospital do SAMS, Portugal; Marta Rebelo, UCIP, Hospital de Egas Moniz, Portugal; Rui Gomes, UCI, Hospital Garcia de Orta, Portugal; Jorge Nunes, Unidade de Cuidados Intensivos, Hospital Lusiadas Lisboa, Portugal; celeste dias, Neurocritical ICU, Hospital de São João, Portugal; Ana Margarida Fernandes, UCI Neurocríticos, Hospital S. José - CHLC EPE, Portugal; Cristina Petrisor, Anaesthesia and Intensive Care 1, Clinical Emergency County Hospital Cluj, Romania; Bodolea Constantin, ATI, Municipal Hospital, Romania; Vladislav Belskiy, Department of Anesthesiology and Intensive Care, Privolzhskiy District Medical Center, Russia; Boris Boskholov, Dept of intensive care, Zhadkevich Clinical Hospital, Russia; Enver Rodriguez, UCI , General Universitario de Castellón, Spain; Sergio Rebollo, ICU, HGU Santa Lucia,Cartagena, Murcia, Spain; Gerardo Aguilar, Unidad de Reanimación - Surgical ICU, Hospital Clínico Universitario de Valencia, Spain; Gaspar Masdeu, Servei Medicina Intensiva, Hospital de Tortosa Verge de la Cinta, Spain; Marián Irazábal Jaimes, Critical Care Unit, Hospital General de Catalunya, Spain; Ángela Prado Mira, Medicina Intensiva, Hospital General Universitario de Albacete, Spain; Maria A. Bodi, General ICU, Hospital Universitari de Tarragona Joan XXIII, Spain; Jesus A. Barea Mendoza, Servicio de Medicina Intensiva, Hospital Universitario 12 de Octubre, Spain; Sonia López-Cuenca, Servicio de Medicina Intensiva y Grandes Quemados, Hospital Universitario de Getafe, Spain; Marcela Homez Guzman, ICU, Hospital Universitario del Henares, Spain; Jesús Rico-Feijoo, Postoperative Critical Care Unit \& Reanimation, Hospital Universitario Río Hortega de Valladolid, Spain; Mercedes Ibarz, ICU Hospital Universitario Sagrado Corazon, Hospital Universitario Sagrado Corazon. Barcelona, Spain; Josep Trenado Alvarez, Intensive Care Department. UCI-Semicritics, Hospital Universitario Mutua Terassa, Spain; Rafael Kawati, central ICU, Akademiska sjukhuset, Sweden; Joakim Sivik, IVA Alingsås Lasarett, Alingsås Lasarett, Sweden; Jessica Nauska, Intensivvårdsavdelning 31, Blekingesjukhuset Karlskrona, Sweden; Daniel Smole, IVA, Centralsjukhuset i Karlstad, Sweden; Fredric Parenmark, IVA, Gävle sjukhus, Sweden; Johanna Lyrén, Intensivvårdsavdelning, Hudiksvalls sjukhus, Gävleborg, Hudiksvalls sjukhus, Sweden; Katalin Rockstroh, IVA, Kalmar Länssjukhus, Sweden; Sara Rydén, Karolinska ICU Huddinge, Karolinska University Hospital Huddinge, Sweden; Martin Spångfors, Intensiven, Kristianstad, Sweden; Morten Strinnholm, ICU Kungälvs Hospital, Kungälvs hospital, Sweden; Sten Walther, Cardiothoracic ICU, Linköping University Hospital, Sweden; Lina De Geer, ICU, Linköping University Hospital, Sweden; Peter Nordlund, OP/IVA Kliniken, Länssjukhuset Ryhov, Sweden; Staffan Pålsson, Intensivvårdsavdelningen, Norrtälje, Sweden; Harald Zetterquist, IVA, Nykopings lasarett, Sweden; Annika Nilsson, IVA, Ornskoldsvik, Ornskoldsviks hospital, Sweden; Karin Thiringer, avdelning 227, IVA, Sahlgrenska University Hospital Mölndal, Sweden; Mårten Jungner, ICU SUS Malmö, Skane University Hospital, Sweden; Björn Bark, IVA Lund, Skåne University Hospital, Sweden; Berit Nordling, IVA Sundsvall, Sundsvall, Sweden; Hans Sköld, ICU, Torsby Sjukhus, Sweden; Camilla Brorsson, CIP, University Hospital Northern Sweden, Sweden; Stefan Persson, Intensivvårsdavdelningen USÖ, University hospital Örebro, Sweden; Anna Bergström, IVA Vrinnevisjukhuset, Vrinnevi hospital, Sweden; Johan Berkius, IVA Västervikssjukhus, Västervikssjukhus, Sweden; Johanna Holmström, Intensivvårdsavdelningen Västerås, Västmanlands sjukhus, Västerås, Sweden; Bernardo Bollen Pinto, Peri-interventional Intermidate Care (SINPI), Geneva University Hospitals, Switzerland; Paul Abraham, Adult Intensive Care (SIA), Geneva University Hospitals, Switzerland; Leila Hergafi, Service des Soins intensifs, Hôpital fribourgeois, Fribourg, Switzerland; Joerg C. Schefold, Universitätsklinik für Intensivmedizin, Inselspital, Bern University Hospital, 
University of Bern, Switzerland; Ewelina Biskup, Medical ICU, University Hospital Basel, Switzerland; I. van Dijk, Intensive Care, Alrijne Ziekenhuis, The Netherlands; L.E.M. van Lelyveld-Haas, Intensive Care, Diakonessenhuis Utrecht, The Netherlands; D.Ramnarain, Intensive Care, Elisabeth Tweesteden Hospital Tilburg, The Netherlands; Tim Jansen, Intensive Care , HagaZiekenhuis, The Netherlands; Fleur Nooteboom, IC LZR, Laurentius Ziekenhuis, The Netherlands; Peter HJ van der Voort, ICU OLVG, OLVG, The Netherlands; Dylan de Lange, Department of Intensive Care Medicine, UMC Utrecht, The Netherlands; Willem Dieperink, Department of Critical Care, University Medical Center Groningen, The Netherlands; Monique C. de Waard, Intensive Care Adults, VU University Medical Center Amsterdam, The Netherlands; Annemarie GE de Smet, Intensive Care Unit, University Medical Centre, University of Groningen, The Netherlands; Laura Bormans, Intensive Care, Zuyderland Medical Centrer, Heerlen, The Netherlands; Tom Dormans, Intensive Care, Zuyderland Medical Center, Heerlen, The Netherlands; Ged Dempsey, Critical Care Unit, Aintree University Hospital NHS Foundation Trust, UK; Shiju J Mathew, ICU, Alexandra Hospital, UK; Ashok S Raj, ICU, Barts Health NHS Trust,Whipps Cross Hospital, UK; Irina Grecu, ITU/HDU, Basingstoke and North Hampshire Hospital, UK; Jason Cupitt, Critical Care Unit, Blackpool Teaching Hospitals NHS Foundation Trust, UK; Tom Lawton, Critical Care Unit, Bradford Royal Infirmary, UK; Richard Clark, ICU, Central Manchester Foundation Trust, UK; Monica Popescu, ICU, Chelsea and Westminster Foundation Trust, West Middlesex University Hospital, UK; Nick Spittle, ICU, Chesterfield Royal Hospital, UK; Maria Faulkner, ICU, Countess of Chester Hospital NHS Foundation Trust, UK; Amanda Cowton, ICU, Darlington memorial Hospital (CDDFT), UK; Esme Elloway, ICU, Derriford Hospital, UK; Patricia Williams, Critical Care Unit, Dorset County Hospital, UK; Michael Reay, Critical Care Unit, Dudley Group of Hospitals NHSFT, Russells Hall Hospital, UK; Srikanth Chukkambotla, Critical Care Unit, East Lancashire Hospitals NHS Trust, UK; Ravi Kumar, CCU, East Surrey Hospital , UK; Nawaf AI-Subaie, ICU, Espsom and St Helier University Hospitals, UK; Linda Kent, Critical Care Unit, Fairfield General Hospital, UK; Tiina Tamm, ICU, Frimley Health, Wexham Park Hospital, UK; Istvan Kajtor, ICU, Frimley Park Hospital, UK; Karen Burns, ICU, Furness General, UK; Richard Pugh, Critical Care Unit, Glan Clwyd Hospital, UK; Marlies Ostermann, ICU, Guys \& St Thomas Hospital, UK; Elisa Kam, ICU, Hillingdon Hospital, UK; Helen Bowyer, Critical Care Centre, Hinchingbrooke Healthcare NHS Trust, UK; Neil Smith, HICU 1\&2, Hull Royal Infirmary, UK; Maie Templeton, Critical Care UNIT, Imperial College Healthcare NHS Trust, UK; Jeremy Henning, ICU2\&3, James Cook Univeristy Hospital, UK; Kelly Goffin, ICU, James Paget University Hospital, UK; Ritoo Kapoor, K\&C ITU, Kent and Canterbury Hospital, UK; Shondipon Laha, CrCU, Lancashire Teaching Hospitals NHS Foundation Trust, UK; Phil Chilton, Critical Care Unit, Leighton Hospital, UK; Waqas Khaliq, ITU/HDU, Lewisham and Greenwich NHS Trust, UK; Alison Crayford, ITU/HDU, Maidstone, UK;
Samantha Coetzee, ICU, Medway NHS Foundation Trust, UK; Moira Tait, Adult ICU, Musgrove Park, UK; Wendy Stoker, ICU, Northumbria Specialist Emergency Care Hospital, UK; Marc Gimenez, ICU, Papworth Hospital NHS Foundation Trust, UK; Alan Pope, Critical Care Unit, Peterborough City Hospital, UK; Julie Camsooksai, Critical Care Unit, Poole Hospital NHS Trust, UK; David Pogson, Dept. of Critical Care, Queen Alexandra Hospital Portsmouth, UK; Kate Quigley, ICU, Queen Elizabeth Hospital, UK; Jenny Ritzema, Critical Care Department, Queen Elizabeth Hospital, Gateshead, UK; Anil Hormis, Critical Care Unit, Rotherham NHS Foundation Trust, UK; Carole Boulanger , ICU, ROYALDEVON\& EXETER NHS FOUNDATION TRUST, UK; M. Balasubramaniam, ICU and HCU, Royal Bolton NHS hospital trust, UK; Luke Vamplew, Critical Care Unit, Royal Bournemouth Hospital, UK; Karen Burt, Critical Care Unit, Royal Cornwall Hospital NHS Trust, UK; Daniel Martin, ICU, Royal Free London NHS Foundation Trust, UK; Irina Grecu, ICU, Royal Hampshire County Hospital, UK; Jayne Craig, ICU, Royal Lancaster Infirmary, UK; John Prowle, Adult Critical Care Unit, Royal London Hospital, UK; Nanci Doyle, ICU, Royal Surrey County Hospital, UK; Jonathon Shelton, Ward 38 ICU, Royal Victoria Infirmary, UK; Carmen Scott, Ward 18 ICU, Royal Victoria Infirmary, UK; Phil Donnison, ICU, Salisbury District Hospital, UK; Sarah Shelton, ICU, Sherwood Forest Hospitals NHS Foundation Trust, UK; Christian Frey, ITU/HDU, South Tyneside District Hospital, UK; Christine Ryan, GICU, St Georges Hospital, UK; Dominic Spray, Cardiothoracic ICU, St Georges Hospital, UK; Christine Ryan, Acute Dependency Unit, St Georges Hospital NHS Trust London, UK; Veronica Barnes, Neuro ICU, St Georges University Hospital NHS Foundation Trust, UK; Kerry Barnes, ITU, st helier hospital, UK; Stephanie Ridgway, Critical Care Unit, NHS Foundation Trust, Tameside General Hospital, UK; Rajnish Saha, Critical Care Unit, The Princess Alexandra NHS Hospital, UK; Linda Kent, ICU, The Royal Oldham Hospital, UK; Thomas Clark, ICU, Torbay Hospital, UK; James Wood, ICU, Tunbridge Wells Hospital, UK; Clare Bolger, General Intensive Care, Univeristy Hospital Southampton NHS Foundation Trust, UK; Christopher Bassford, General Critical Care, University Hospital Coventry, UK; Amanda Cowton, ICU, University hospital of North Durham, UK; john lewandowski, Critical Care Unit, University Hospital of North Tees, UK; Xiaobei Zhao, ICU (Level 6), Watford General Hospital / West Hertfortshire NHS trust, UK; Sally Humphreys, Critical Care, West Suffolk NHS Foundation Trust, UK; Susan Dowling, Ward 4E Critical Care unit, Whiston, UK; Neil Richardson, ICU, William Harvey Hospital, Ashford, UK; Andrew Burtenshaw, Critical Care Unit, Worcestershire Royal Hospital, UK; Carl Stevenson, ICU, Wye Valley NHS Trust, UK; Danielle Wilcock, Critical Care Unit, York Teaching Hospital NHS Foundation Trust, UK; Yuiry Nalapko, Anaesthesia and Intensive Care, Lugansk State Medical University, Ukraine.

\section{Published online: 20 August 2018}

